\title{
Glycolysis determines dichotomous regulation of T cell subsets in hypoxia
}

\author{
Yang Xu, ${ }^{1,2}$ Arindam Chaudhury, ${ }^{3}$ Ming Zhang, ${ }^{1}$ Barbara Savoldo, ${ }^{1,4}$ Leonid S. Metelitsa, ${ }^{2,4}$ John Rodgers, ${ }^{2}$ Jason T. Yustein, ${ }^{4}$ \\ Joel R. Neilson, ${ }^{3}$ and Gianpietro Dotti ${ }^{1,2}$ \\ 'Center for Cell and Cene Therapy, ${ }^{2}$ Department of Pathology and Immunology, ${ }^{3}$ Department of Molecular Physiology and Biophysics and Dan L. Duncan Cancer Center, and ${ }^{4}$ Department of Pediatrics, \\ Texas Children's Cancer Center, Baylor College of Medicine, Houston, Texas, USA.
}

\begin{abstract}
Hypoxia occurs in many pathological conditions, including chronic inflammation and tumors, and is considered to be an inhibitor of T cell function. However, robust T cell responses occur at many hypoxic inflammatory sites, suggesting that functions of some subsets are stimulated under low oxygen conditions. Here, we investigated how hypoxic conditions influence human $T$ cell functions and found that, in contrast to naive and central memory $T$ cells $\left(T_{N}\right.$ and $T_{C M}$ ), hypoxia enhances the proliferation, viability, and cytotoxic action of effector memory $T$ cells $\left(T_{E M}\right)$. Enhanced TEM expansion in hypoxia corresponded to high hypoxia-inducible factor $1 \alpha$ (HIF1 $\alpha$ ) expression and glycolytic activity compared with that observed in $T_{N}$ and $T_{C M}$. We determined that the glycolytic enzyme GAPDH negatively regulates HIF1A expression by binding to adenylate-uridylate-rich elements in the $3^{\prime}$-UTR region of HIF1A mRNA in glycolytically inactive $\mathrm{T}_{\mathrm{N}}$ and $\mathrm{T}_{\mathrm{CM}}$. Conversely, active glycolysis with decreased CAPDH availability in $\mathrm{T}_{\mathrm{EM}}$ resulted in elevated HIF1 $\alpha$ expression. Furthermore, CAPDH overexpression reduced HIF1 $\alpha$ expression and impaired proliferation and survival of T cells in hypoxia, indicating that high glycolytic metabolism drives increases in HIF1 $\alpha$ to enhance $\mathrm{T}_{\mathrm{EM}}$ function during hypoxia. This work demonstrates that glycolytic metabolism regulates the translation of HIF1A to determine T cell responses to hypoxia and implicates GAPDH as a potential mechanism for controlling $T$ cell function in peripheral tissue.
\end{abstract}

\section{Introduction}

$\mathrm{T}$ cells experience a broad range of $\mathrm{O}_{2}$ tension in vivo, varying from $13 \%$ in peripheral arterial blood (1) to $5 \%$ in normal tissues with increased distance from blood vessels (2), to less than $2 \%$ in chronically inflamed tissues (3) and solid tumor microenvironments (4). Local $\mathrm{O}_{2}$ tension is an environmental factor that affects $\mathrm{T}$ cell function $(5,6)$. In particular, low $\mathrm{O}_{2}$ tension $\left(1 \% \mathrm{O}_{2}\right.$; hypoxia) impairs the proliferation of human peripheral blood $\mathrm{T}$ cells in vitro and the activation of mouse splenic T cells in vivo $(7,8)$. However, the inhibitory effects that hypoxia is thought to have on $\mathrm{T}$ cells are inconsistent with the robust expansion of $\mathrm{T}$ cells in many hypoxic inflammatory sites (9-11). Recent studies demonstrate that hypoxia-related pathways can facilitate the differentiation of $\mathrm{CD}^{+}$cytotoxic $\mathrm{T}$ lymphocytes (CTLs) (12) and clearance of chronic viral infection and tumors (13). These new findings suggest that low $\mathrm{O}_{2}$ tension in tissues can be inhibitory for certain $\mathrm{T}$ cell subsets, but stimulatory for other $\mathrm{T}$ cell subsets that must be functional in hypoxic inflamed or neoplastic tissues. For instance, circulating $\mathrm{T}$ cells and those located in secondary lymphoid organs are mainly naive cells $\left(\mathrm{T}_{\mathrm{N}}\right)$ and central memory

Note regarding evaluation of this manuscript: Manuscripts authored by scientists associated with Duke University, The University of North Carolina at Chapel Hill, Duke-NUS, and the Sanford-Burnham Medical Research Institute are handled not by members of the editorial board but rather by the science editors, who consult with selected external editors and reviewers.

Conflict of interest: The authors have declared that no conflict of interest exists. Submitted: December 7, 2015; Accepted: April 27, 2016.

Reference information: J Clin Invest. 2016;126(7):2678-2688. doi:10.1172/JCI85834.
T cells $\left(\mathrm{T}_{\mathrm{CM}}\right)$, while $\mathrm{T}$ cells in peripheral tissues in pathologic conditions such as inflammation or tumors are predominantly effector memory $\mathrm{T}$ cells $\left(\mathrm{T}_{\mathrm{EM}}\right)$ and effector $\mathrm{T}$ cells $\left(\mathrm{T}_{\mathrm{E}}\right)(14)$. Whether the low $\mathrm{O}_{2}$ tension has distinct effects on $\mathrm{T}$ cell memory subsets that are differentially located within tissues remains unknown.

Hypoxia-inducible factors (HIFs) are transcription factors that facilitate cellular responses to hypoxia. HIFs are heterodimeric proteins consisting of $\alpha$ (HIF1 $\alpha$, HIF $2 \alpha$, and HIF $3 \alpha)$ and $\beta$ (HIF1 $\beta$ ) subunits. While the $\beta$ subunit is constitutively expressed, the $\alpha$ subunits are dynamically regulated by various mechanisms (2). In normoxia, the $\alpha$ subunits undergo $\mathrm{O}_{2}$-dependent hydroxylation and proteosomal degradation via the $\mathrm{E} 3$ ligase von Hippel Lindau (VHL) complex (15). By contrast, $\alpha$ subunits are stabilized under conditions of low $\mathrm{O}_{2}$ tension (15) or genetic deletion of VHL (13). In T cells, HIF1 $\alpha$ expression is also induced both transcriptionally and translationally by $\mathrm{T}$ cell receptor (TCR) stimulation $(16,17)$, which drives glycolytic metabolism by transcriptionally activating enzymes involved in glycolysis $(12,18)$. Increased glycolysis mediated by HIF1 resembles the "metabolic switch" occurring during $\mathrm{T}$ cell activation $(17,19)$ : while resting $\mathrm{T}$ cells primarily use oxidative phosphorylation (OXPHOS) to generate ATP, activated $\mathrm{T}$ cells reprogram the metabolism to favor glycolysis to fulfill the bioenergetic and biosynthetic requirement for rapid proliferation, even when oxygen is available for OXPHOS (17). Because the HIF1 pathway is active during $\mathrm{T}$ cell stimulation $(13,17,18)$, the hypoxia/ HIF1-facilitated glycolysis may converge in activated $\mathrm{T}$ cells with endogenous glycolytic induction to synergistically support the proliferative and effector functions. 
A
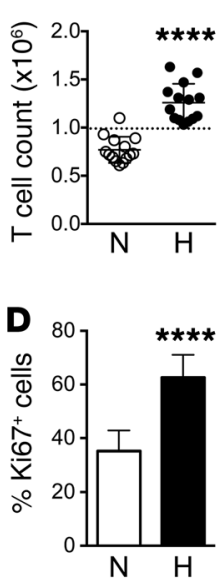

$\mathbf{F}$

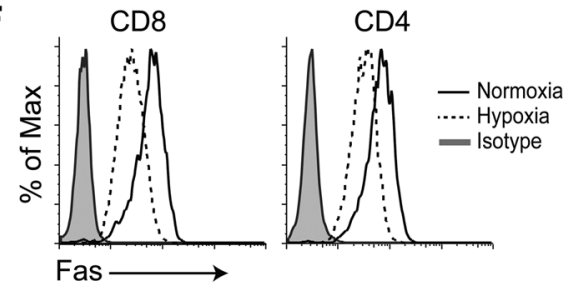

B

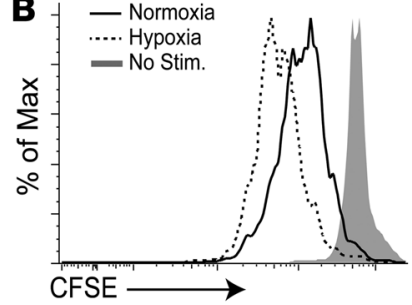

E

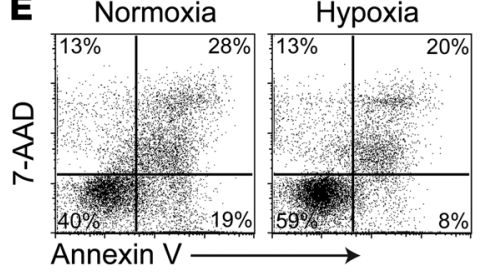

C
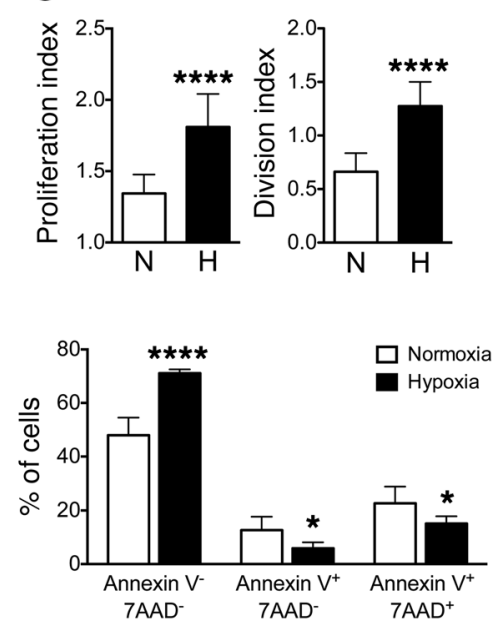

H
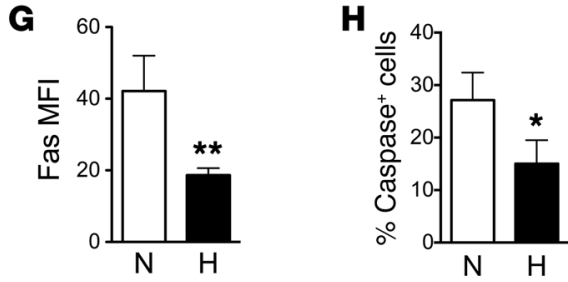

Figure 1. Proliferation and survival of human $\mathrm{T}_{\mathrm{EXP}}$ are enhanced in hypoxia. $\mathrm{T}_{\mathrm{EXP}}$ were activated with OKT3/a-CD28 Abs in either normoxia (N) or hypoxia (H). (A) Cell counts of $\mathrm{T}_{\text {EXP }} 72$ hours after activation. $n=14$. ${ }^{* * * * P}<0.0001$, paired Student's $t$ test. (B) CFSE dilution of CFSE-labeled $\mathrm{T}_{\text {EXP }} 72$ hours after activation. (C) Quantitative analysis of the CFSE dilution in B. Proliferation and division indexes were calculated using Flowjo software. $n=10$. ${ }^{* * *} P<$ 0.0001 , paired Student's $t$ test. (D) Expression of Ki67 in $T_{\text {ExP }} 72$ hours after activation. $n=8$. ${ }^{* * *} P<0.001$, paired Student's $t$ test. (E) Annexin $V$ and 7-AAD staining of $\mathrm{T}_{\text {EXP }} 72$ hours after activation. $n=6 .{ }^{* * * *} P<0.0001,{ }^{*} P=0.029$ for annexin $\mathrm{V}^{+} / 7-\mathrm{AAD}^{-}$cell population, and $P=0.015$ for annexin $\mathrm{V}^{+} / 7-$ $A A D^{+}$cell population, 2-way ANOVA with Bonferroni's post-hoc analysis. ( $F$ and $\mathbf{G}$ ) Surface expression of CD95/Fas in $T_{\text {EXP }} 48$ hours after activation. $n=6$. ${ }^{*} P=0.0016$, paired Student's $t$ test. (H) Percentage of caspase ${ }^{+}$cells in live $T_{\text {Exp }} 72$ hours after activation. Live cells were gated on the basis of forward scatter (FSC) and side scatter (SSC). $n=3 .{ }^{*} P=0.0152$, paired Student's $t$ test. Error bars indicate SD. Max, maximum.

Here, we show that $\mathrm{T}_{\mathrm{EM}}$ have immediate superior proliferation and effector function under hypoxic conditions, while $\mathrm{T}_{\mathrm{N}}$ and $\mathrm{T}_{\mathrm{CM}}$ are inhibited under these conditions. This distinct pattern of hypoxia response is attributed to the differential expression of HIF1 $\alpha$ and related glycolytic activity in $\mathrm{T}$ cell memory subsets. Furthermore, the differential HIF1 $\alpha$ expression is linked to a novel mechanism of translational regulation by the glycolytic enzyme GAPDH in T cells.

\section{Results}

Proliferation and survival of human $T_{E M}$ are enhanced in hypoxia. Previous studies have suggested an inhibitory role of hypoxia in activated human $T$ cells (20). We confirmed that freshly isolated peripheral blood $\mathrm{T}$ cells ( $\mathrm{PB}-\mathrm{Ts}$ ) have impaired proliferation and viability upon activation with OKT3/a-CD28 Abs in hypoxia (1\% $\mathrm{O}_{2}$ ) as compared with that observed in normoxia $\left(20 \% \mathrm{O}_{2}\right)$ (Supplemental Figure 1; supplemental material available online with this article; doi:10.1172/JCI85834DS1). However, unselected PB-Ts predominantly consist of $\mathrm{T}$ cells with a $\mathrm{T}_{\mathrm{N}}\left(\mathrm{CD} 45 \mathrm{RA}^{+} \mathrm{CCR} 7^{+}\right)$or $\mathrm{T}_{\mathrm{CM}}\left(\mathrm{CD}^{2} 5 \mathrm{RA}^{-} \mathrm{CCR}^{+}\right)$phenotype, with the $\mathrm{T}_{\mathrm{EM}}$ (CD45RA-CCR7 ${ }^{-}$) subset underrepresented in PB-Ts (Supplemental Figure 2, A and B). Additionally, because $\mathrm{T}_{\mathrm{N}}$ and $\mathrm{T}_{\mathrm{CM}}$ show higher intrinsic proliferative potential upon antigen stimulation than do $\mathrm{T}_{\mathrm{EM}}$ (14), any specific effect of hypoxia in $\mathrm{T}_{\mathrm{EM}}$ could be underrepresented when analyzing unfractionated $\mathrm{PB}-\mathrm{Ts}$, given the predominance of $\mathrm{T}_{\mathrm{N}}$ and $\mathrm{T}_{\mathrm{CM}}$. Therefore, we aimed to specifically study $\mathrm{T}_{\mathrm{EM}}$ upon activation in hypoxia. To acquire sufficient numbers of $\mathrm{T}_{\mathrm{EM}}$, we activated human peripheral blood mononuclear cells (PBMCs) with OKT3/a-CD28 Abs and cultured them with IL-2 for 12 days. These ex vivo-expanded $\mathrm{T}$ cells $\left(\mathrm{T}_{\mathrm{EXP}}\right)$ were enriched in CD45RA-CCR7 ${ }^{-}$ cells $(62.8 \% \pm 3.1 \%$ vs. $12.4 \% \pm 2.5 \%$ in $\mathrm{PB}-\mathrm{Ts})$ and were phenotypically similar to the $\mathrm{T}_{\mathrm{EM}}$ detected in $\mathrm{PB}-\mathrm{Ts}$, based on various $\mathrm{T}$ cell differentiation markers (CD25, CD27, CD28, CD45RO, and CD62L) (Supplemental Figure 2). We activated $\mathrm{T}_{\mathrm{EXP}}$ with OKT3/aCD28 Abs under hypoxic or normoxic conditions and measured proliferation and cell viability 72 hours after stimulation. In sharp contrast to unselected PB-Ts, cell counts of $\mathrm{T}_{\mathrm{EXP}}$ were higher in hypoxia than in normoxia (Figure 1A) as a result of both enhanced cell division (Figure 1, B-D) and reduced cell apoptosis (Figure 1E). In hypoxia, $\mathrm{T}_{\mathrm{EXP}}$ showed lower expression of the death receptor CD95/Fas (Figure 1, F and G) and reduced caspase activity (Figure $1 \mathrm{H}$ ), which further suggests a reduced potential for induction of apoptosis. In addition, mRNA expression of the antiapoptotic genes BCL2 and BNIP3 was significantly higher in $\mathrm{T}_{\text {EXP }}$ activated in hypoxia, while the expression of FAS mRNA decreased (Supplemental Figure 3), correlating with the reduced apoptosis of $\mathrm{T}_{\text {EXP }}$ in hypoxia. $\mathrm{T}_{\mathrm{EXP}}$ showed a comparable percentage of $\mathrm{CD} 4^{+}$ and $\mathrm{CD} 8^{+} \mathrm{T}$ cells when activated in hypoxia or normoxia (Supplemental Figure 4A). Furthermore, both $\mathrm{CD}^{+}$and $\mathrm{CD} 8^{+} \mathrm{T}_{\mathrm{EXP}}$ dis- 

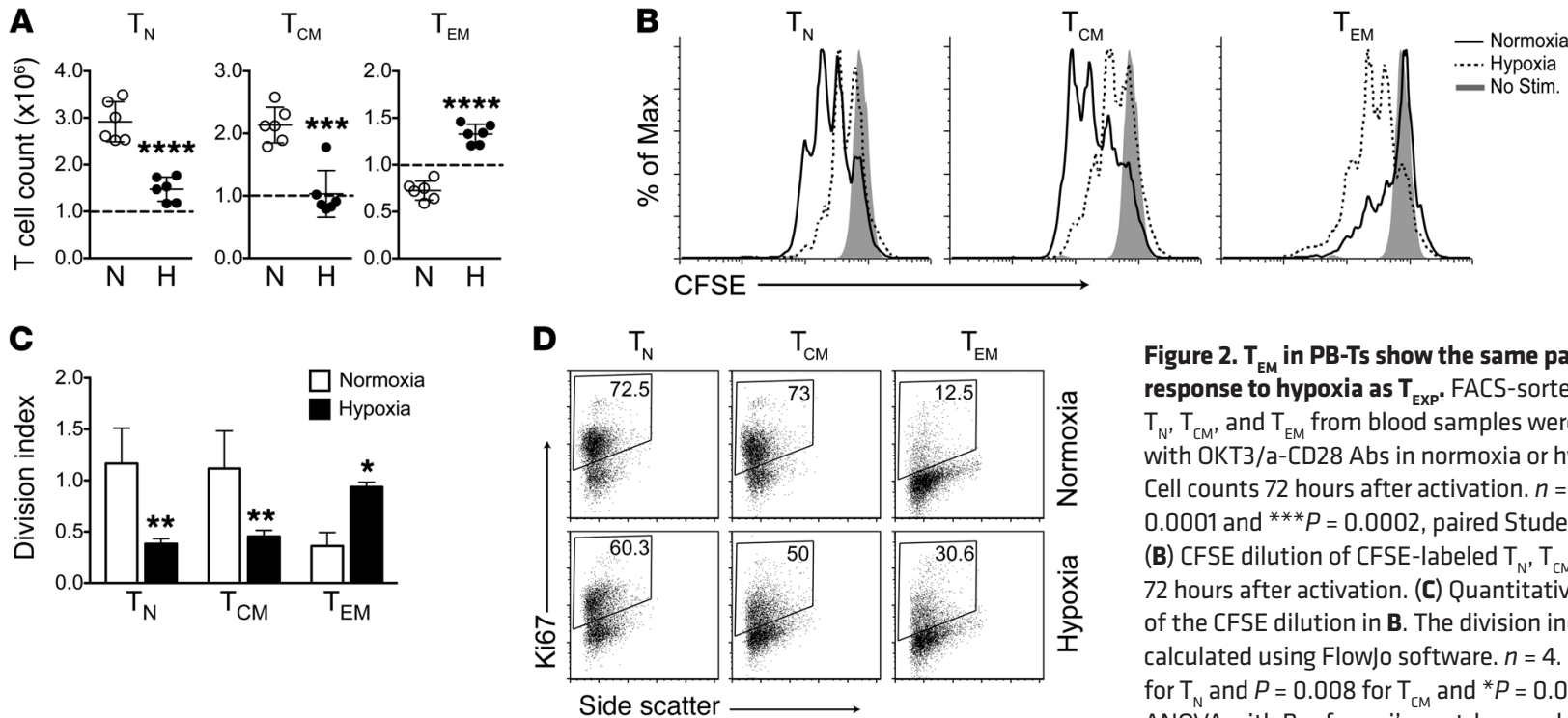

\begin{abstract}
Figure 2. $\mathrm{T}_{E M}$ in PB-Ts show the same pattern of response to hypoxia as $T_{\text {EXP. }}$. FACS-sorted human $T_{N}, T_{C M}$, and $T_{E M}$ from blood samples were activated with 0 KT3/a-CD28 Abs in normoxia or hypoxia. (A) Cell counts 72 hours after activation. $n=6 .{ }^{* * *} P<$ 0.0001 and ${ }^{* * *} P=0.0002$, paired Student's $t$ test. (B) CFSE dilution of CFSE-labeled $T_{N}, T_{C M}$, and $T_{E M}$ 72 hours after activation. (C) Quantitative analysis of the CFSE dilution in $\mathbf{B}$. The division index was calculated using Flowjo software. $n=4 .{ }^{* *} P=0.003$ for $\mathrm{T}_{\mathrm{N}}$ and $P=0.008$ for $\mathrm{T}_{\mathrm{CM}}$ and ${ }^{*} P=0.018$, 2-way ANOVA with Bonferroni's post-hoc analysis. (D) Expression of Ki67 in $T_{N}, T_{C M}$, and $T_{E M} 72$ hours after activation. $n=3$. (E) Percentage of live $T_{N}, T_{C M}$, and $\mathrm{T}_{\mathrm{EM}} 72$ hours after activation. $n=6 .{ }^{* * *} P<0.0001$, 2-way ANOVA with Bonferroni's post-hoc analysis. (F) Expression of CD95/Fas in $\mathrm{T}_{\mathrm{N}}, \mathrm{T}_{\mathrm{CM}}$, and $\mathrm{T}_{\mathrm{EM}} 48$ hours after activation. $n=3 .{ }^{* *} P=0.0011,2$-way ANOVA with Bonferroni's post-hoc analysis. Error

bars indicate SD. MFI, mean fluorescence intensity.
\end{abstract}
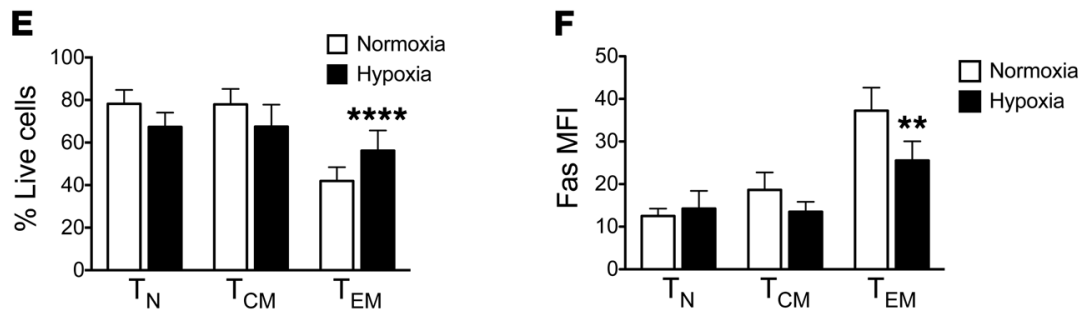

played enhanced proliferation and survival in hypoxia compared with that observed in normoxia (Supplemental Figure 4, B-D). These results suggest that the dichotomous influence of hypoxia on $\mathrm{PB}-\mathrm{Ts}$ versus that on $\mathrm{T}_{\mathrm{EXP}}$ was not due to the disparities in CD4 and CD8 composition. Finally, hypoxia did not affect the production of IL-2, IFN- $\gamma$, or TNF- $\alpha$, nor did it affect the expression of the IL-2 receptor $\mathrm{CD} 25$ or the early $\mathrm{T}$ cell activation marker CD69 (Supplemental Figure 4, E and F). Overall, these data suggest that $\mathrm{T}_{\mathrm{EXP}}$ show a unique pattern of response upon activation in hypoxia.

Because $\mathrm{T}_{\mathrm{EXP}}$ are generated through ex vivo culture, which may alter the native characteristics of these cells, even if they are phenotypically similar to the $\mathrm{T}_{\mathrm{EM}}$ detected in the PB-Ts, we sorted $\mathrm{T}_{\mathrm{N}}, \mathrm{T}_{\mathrm{CM}}$, and $T_{E M}$ from PB-Ts on the basis of the expression of CD45RA and CCR7 (Supplemental Figure 5 and ref. 21). These cells were then stimulated with OKT3/a-CD28 Abs in hypoxia or normoxia for 72 hours without any intermediate step of ex vivo culture. Consistent with the results obtained with $\mathrm{T}_{\mathrm{EXP}}$, the $\mathrm{T}_{\mathrm{EM}}$ displayed superior numeric expansion under hypoxic conditions compared with that observed under normoxic conditions (Figure 2A), with a corresponding enhancement of cell division (Figure 2, B-D) and reduced cell death (Figure 2, E and F). By contrast, hypoxia impaired the expansion and cell division of $\mathrm{T}_{\mathrm{N}}$ and $\mathrm{T}_{\mathrm{CM}}$ (Figure 2, $\mathrm{A}-\mathrm{C}$ ), which is likely to account for the observed overall inhibition of proliferation of unselected $\mathrm{PB}-\mathrm{Ts}$ (Supplemental Figure 1). Notably, while CD95/Fas expression in $\mathrm{T}_{\mathrm{N}}$ and $\mathrm{T}_{\mathrm{CM}}$ was comparable under hypoxic and normoxic conditions, the level of expression in these subsets was 2- to 3-fold lower than that detected in $\mathrm{T}_{\mathrm{EM}}$ (Figure $2 \mathrm{~F}$ ), which is consistent with the reduced susceptibility of $\mathrm{T}_{\mathrm{N}}$ and $\mathrm{T}_{\mathrm{CM}}$ to Fas-mediated apoptosis (22). Hence, the reduced expansion of $\mathrm{T}_{\mathrm{N}}$ and $\mathrm{T}_{\mathrm{CM}}$ in hypoxia is likely due to reduced cell division. Collectively, these data indicate that $\mathrm{T}_{\mathrm{EXP}}$ and $\mathrm{T}_{\mathrm{EM}}$ are intrinsically equipped to positively respond to hypoxia, a finding that functionally distinguishes them from $\mathrm{T}_{\mathrm{N}}$ and $\mathrm{T}_{\mathrm{CM}}$.

Cytotoxic function of tumor-specific $T_{E X P}$ is enhanced by hypoxia. Because hypoxia positively regulates the proliferation and survival of $\mathrm{T}_{\mathrm{EM}}$, we speculated that hypoxia might also modulate their cytotoxic function. To confer antigen specificity to $\mathrm{T}_{\mathrm{EM}}$, we transduced activated human $\mathrm{T}$ cells with a retroviral vector encoding a GD2-specific chimeric antigen receptor (CAR.GD2-T) (23) and cocultured CAR.GD2-T with GD2 ${ }^{+}$neuroblastoma tumor cells (LA$\mathrm{N}-1)$ in normoxia or hypoxia for 48 hours. Fewer tumor cells survived when cocultured with CAR.GD2-T in hypoxia than occurred in normoxia. That this was a result of cytotoxicity rather than impaired tumor cell growth in hypoxic conditions was illustrated by coculture of tumor cells with nontransduced T cells (NTs), in which a similar percentage and number of surviving tumor cells were observed in both hypoxic and normoxic conditions at the end of the experiment (Figure 3, A and B). Although $\mathrm{T}_{\mathrm{EXP}}$ showed enhanced cell expansion in hypoxia at 72 hours upon OKT3/a-CD28 stimulation, after 48 hours of coculture, the number of CAR.GD2-T was similar in hypoxic and normoxic conditions (Figure 3C). To determine whether the observed enhanced antitumor activity in hypoxia is due to altered tumor cell susceptibility to the cytotoxic activity of CAR.GD2-T, we used LA-N-1 cells cultured in hypoxia or normoxia as target cells in cytotoxicity assays performed in normoxia. LA-N-1 cells cultured in hypoxia did not show increased susceptibility to lysis by CAR.GD2-T compared with that observed with LA-N-1 cells growing in normoxic conditions (Figure 3D). In addition, we observed a similar CD4/CD8 composition of CAR. 
A

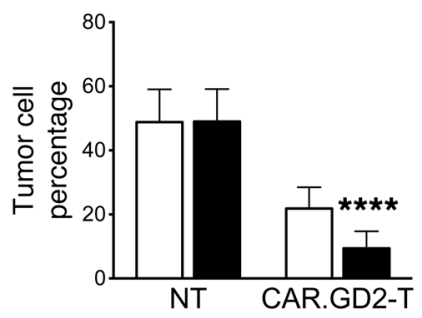

C CAR.GD2-T

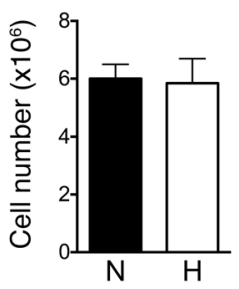

$\mathbf{F}$

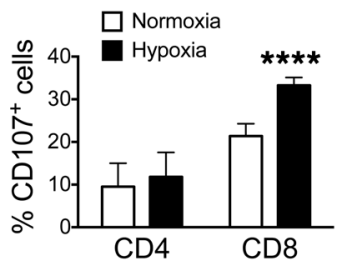

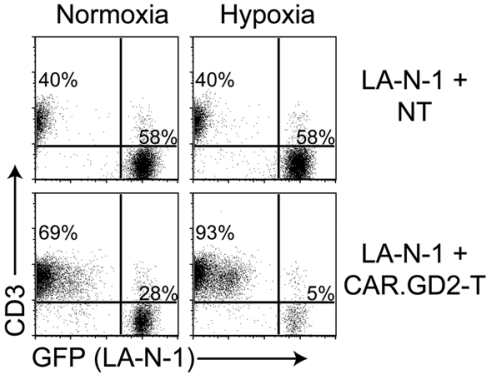

D
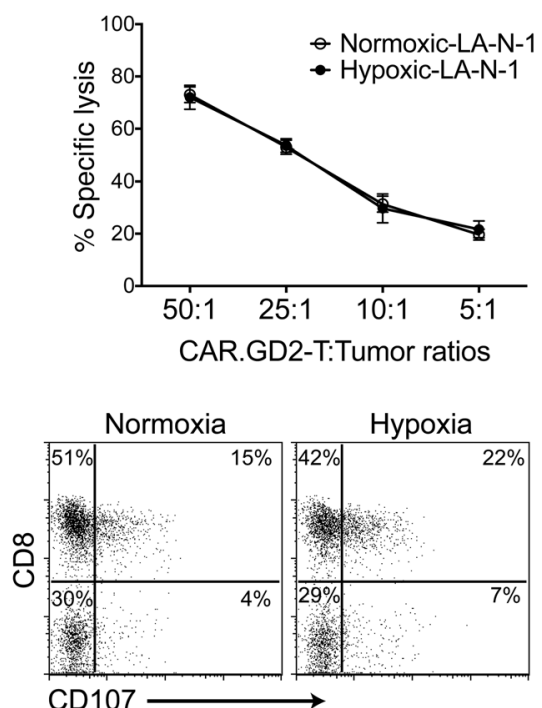

B

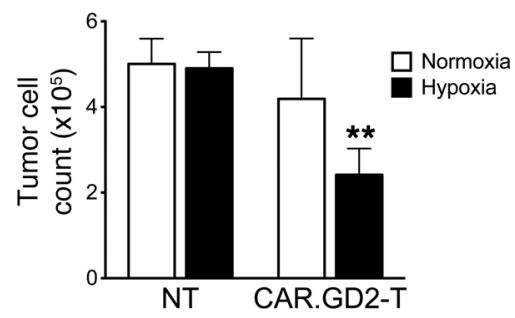

E

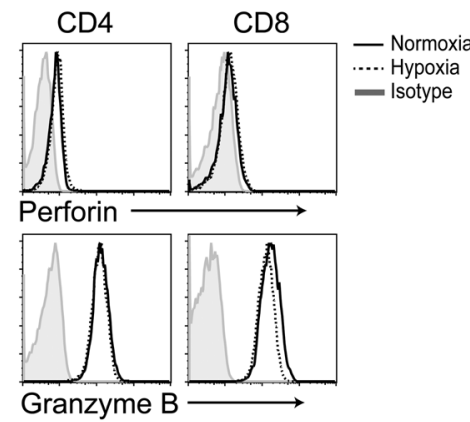

G

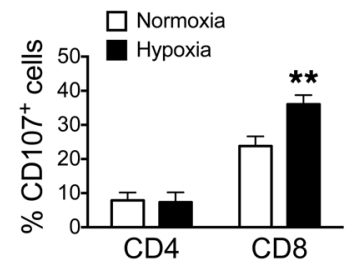

Figure 3. The cytotoxic function of CAR-redirected $\mathrm{T}_{\mathrm{EXP}}$ is superior in hypoxia. CAR.GD2-T were cocultured with LA-N-1 GFP+ neuroblastoma cells at a 1:4 ratio for 48 hours in normoxia or hypoxia. NTs were used as negative cytotoxic controls. (A-C) The percentage and number of GFP+CD3- tumor cells and number of GFP-CD3+ CAR.GD2-T were determined by flow cytometry after 48 hours of coculture. The percentages in the upper left and bottom right quadrants in $\mathbf{A}$ indicate the percentage of T cells and tumor cells, respectively. $n=8$ (A, left) and $n=4$ (B and $\mathbf{C})$. ${ }^{* * *} P<0.0001(\mathbf{A})$ and ${ }^{* *} P=0.004(\mathbf{B})$, 2-way ANOVA with Bonferroni's post-hoc analysis. (D) LA-N-1 GFP+ cells were precultured in hypoxia or normoxia for 48 hours, labeled with ${ }^{51} \mathrm{Cr}$, and cocultured with CAR.GD2-T in normoxia at E:T ratios of 50:1, 25:1, 10:1, and 5:1. The cytotoxic activity of CAR.GD2-T was determined after 4 hours of coculture. $n=3$. (E) Expression of perforin and granzyme B in CAR.CD2-T stimulated with the anti-CAR idiotype Ab (1A7) for 24 or 48 hours in normoxia or hypoxia. $n=3$. (F and $\mathbf{G}$ ) Surface expression of CD107a/b in CAR.CD2-T stimulated with either 1A7 Ab (F) or OKT3/a-CD28 (G) Abs for 6 hours. The percentages in the upper right and bottom right quadrants in $\mathbf{F}$ indicate the percentage of degranulated CD8 and CD4 T cells, respectively. $n=3$. ${ }^{* *} P=0.0059$ and ${ }^{* * * *} P<0.0001,2$-way ANOVA with Bonferroni's post-hoc analysis. Error bars indicate SD.

GD2-T after a 2-day coculture in normoxic and hypoxic conditions (Supplemental Figure 6A), while CAR.GD2-T displayed superior cytotoxicity against LA-N-1 cells in hypoxic conditions compared with that observed in normoxic conditions in a ${ }^{51} \mathrm{Cr}$ release assay (Supplemental Figure 6B). Collectively, these data suggest an increase in the per-cell cytotoxicity of CAR.GD2-T cells in hypoxia.

Unlike mouse CD8 $8^{+}$CTLs, which express increased levels of lytic enzymes in hypoxia $(12,13)$, the expression of perforin and granzyme $B$ in activated human CAR.GD2-T remained unchanged in hypoxia (Figure 3E). However, the superior antitumor activity of CAR.GD2-T in hypoxia correlated with enhanced degranulation of $\mathrm{CD} 8^{+}$CAR.GD2-T upon specific CAR.GD2 activation (Figure 3F). Stimulation of CAR.GD2-T in hypoxia with OKT3/a-CD28 Abs also induced more degranulation (Figure 3G), indicating that the enhanced degranulation in hypoxia is not due to an intrinsic property of the CAR.GD2 molecule but is recapitulated upon TCR stimulation. Collectively, our data indicate that the cytotoxic function of $\mathrm{T}_{\mathrm{EXP}}$ is enhanced under hypoxic conditions.
Tumor-related hypoxia promotes the cycling of tumor-specific $T_{E X P}$ in vivo. Solid tumors are well recognized as hypoxic tissues, with $\mathrm{O}_{2}$ tension ranging from $0 \%$ to $4 \%$, depending on the distance from the blood vessel $(4,24,25)$. Previous studies have indicated that tumor-infiltrating $\mathrm{T}$ cells are present in both $\mathrm{O}_{2}$-rich and $\mathrm{O}_{2}$-poor areas of tumor $(26,27)$. We thus used xenograft tumor models to determine whether low $\mathrm{O}_{2}$ tension within the tumor affects the proliferation of $\mathrm{T}_{\mathrm{EM}}$ in vivo. We engrafted NOD/SCID $/ \gamma_{\mathrm{c}}{ }^{-1-}$ (NSG) mice s.c. with neuroblastoma tumor cells. After engraftment, we intratumorally inoculated the mice with CAR.GD2-T or control $\mathrm{T}_{\mathrm{EXP}}$ expressing a nontumor-targeting CAR specific for the CD19 antigen (CAR.CD19-T) (Figure 4A). The hypoxia-labeling chemical pimonidazole (hydroxyprobe [HP1]) $(28,29)$ specifically labeled in vivo CAR.GD2-T located in either hypoxic $\left(\mathrm{O}_{2}<1.3 \% \mathrm{HP}^{+}\right)$or normoxic $\left(\mathrm{O}_{2}>1.3 \% \mathrm{HP}^{-}\right)$tumor areas (ref. 28 and Figure $\left.4 \mathrm{~B}\right)$. Strikingly, as assessed by expression of the mitotic marker Ki67, CAR. GD2-T were characterized by greater proliferation within $\mathrm{O}_{2}$-poor tumor areas $\left(\mathrm{HP}^{+}\right)$when compared with those in $\mathrm{O}_{2}$-rich areas 
A

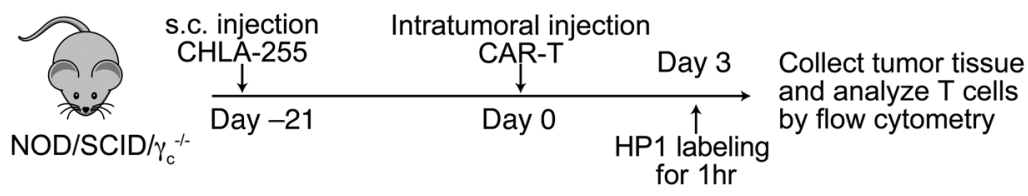

C

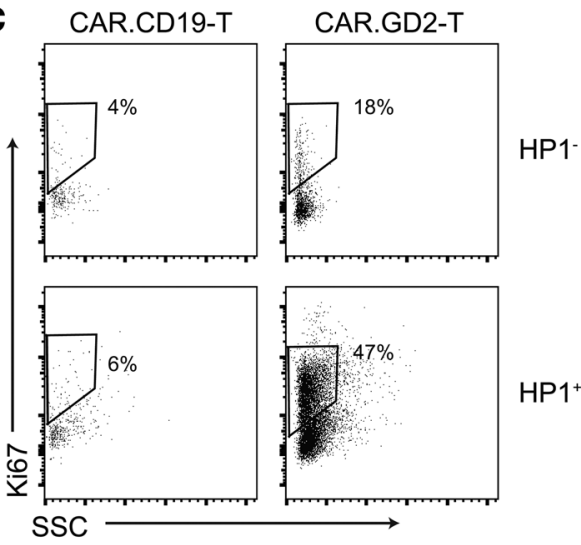

D

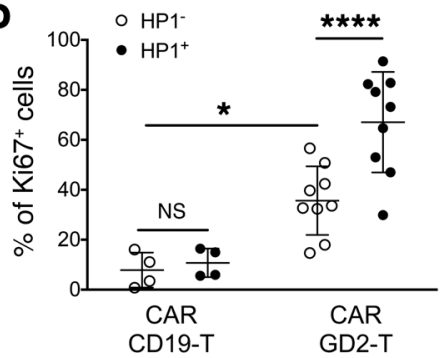

$\mathrm{HP}^{+}$

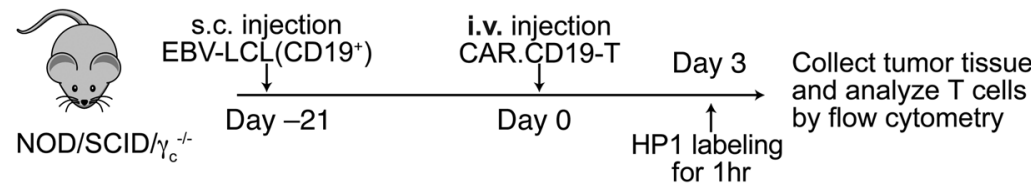

B
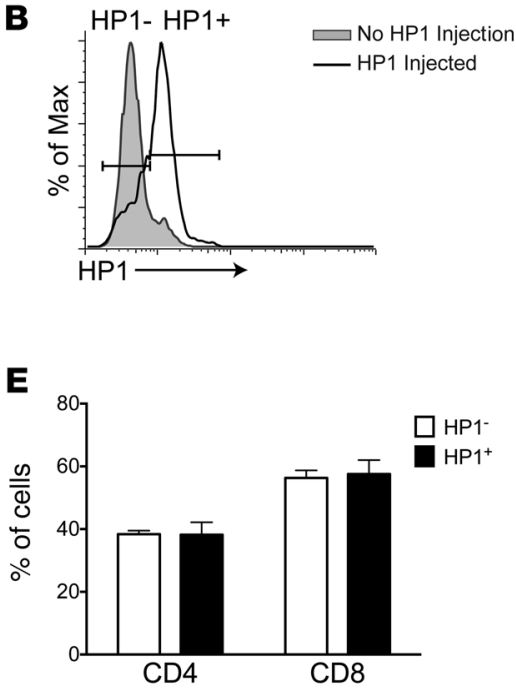

G

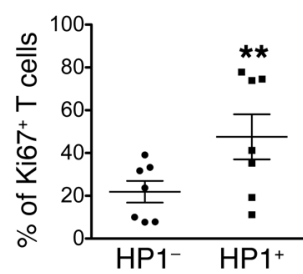

Figure 4. CAR-redirected $T_{\text {EXP }}$ show enhanced cycling in hypoxic tumors in vivo. (A) CHLA-255 neuroblastoma cells were engrafted s.c into NSG mice. By day 21, either CAR.CD2-T or CAR.CD19-T were injected intratumorally, and 3 days later, mice were inoculated with the hypoxia-labeling chemical HP1. Tumors were removed from mice 1 hour after the inoculation of HP1. (B) Intratumoral CAR-Ts (CD45+CD3+) were stained with Abs specific to HP1. T cells from a mouse without HP1 injection (gray) were used as a negative control for gating HP1+ or HP1- $\mathrm{T}$ cells. (C and D) Percentage of Ki67 ${ }^{+}$cells in $\mathrm{HP}^{+}{ }^{+}$or $\mathrm{HP}^{-}$ tumor-specific CAR.GD2-T or nontumor-targeting CAR.CD19-T. $n=8$ for CAR.CD2-T and $n=4$ for CAR.CD19-T. The percentages in C indicate the percentage of proliferating cells. ${ }^{*} P=0.0112$ and ${ }^{* * *} P<0.0001$, 2-way ANOVA with Bonferroni's post-hoc analysis. (E) Percentage of CD4 ${ }^{+}$or CD8 ${ }^{+}$CAR.CD2-T in $\mathrm{HP1}^{-}$or $\mathrm{HP}^{+}$cell populations. $n=3$. (F) EBV-transformed B cells (EBV-LCL) were engrafted s.c. into NSG mice. By day 21, CAR.CD19-T were injected i.v., and 3 days later, mice were inoculated with HP1. Tumors were removed from the mice 1 hour after the HP1 inoculation. (G) Percentage of Ki67 ${ }^{+}$cells in $\mathrm{HP}^{+}$or HP1- CAR.CD19-T. $n=7$. ${ }^{* *} P=0.0053$, paired Student's $t$ test. Error bars indicate SD.

(HP1'). This effect was antigen specific, as CAR.CD19-T showed minimal proliferation in both $\mathrm{HP}^{+}$and $\mathrm{HP}^{-}$areas (Figure $4, \mathrm{C}$ and D). Of note, the percentages of $\mathrm{CD}^{+}$and $\mathrm{CD} 8^{+}$CAR.GD2-T were comparable in $\mathrm{HP}^{-}$and $\mathrm{HP}^{+}$areas (Figure 4E), suggesting that there is no preferential localization or expansion of either population in hypoxic tumor areas. In a second tumor model in which mice were engrafted s.c. with $\mathrm{CD} 19^{+}$lymphoma cells and then infused i.v. with CAR.CD19-T, we also found that the CAR.CD19-T that reached hypoxic tumor areas were more proliferative than those that reached normoxic tumor areas (Figure 4, F and G). Collectively, these in vivo experiments indicate that hypoxia enhances the proliferation of antigen-specific CAR-T.

$T_{\text {EXP }}$ display elevated HIF1 $\alpha$ expression and glycolytic activity. HIF1 is a critical transcriptional factor that facilitates cellular adaptation to hypoxia $(2,30)$. To assess whether HIF1 plays a role in $\mathrm{T}_{\mathrm{EXP}}$ function within our experimental model, we blocked its activity with the HIF1 $\alpha$ inhibitor BAY87-2243 (31) or overexpressed HIF1 $\alpha$ via chemical mimicking of hypoxia (dimethyloxaloylglycine [DMOG]) (32) or gene transfer. We found that HIF1 $\alpha$ was required and sufficient for promoting the proliferation of $\mathrm{T}_{\mathrm{EXP}}$ (Supplemental Figure 7, $\mathrm{A}-\mathrm{F}$ ), which highlights the critical role of HIF1 $\alpha$ in $\mathrm{T}_{\mathrm{EXP}}$ under hypoxic conditions. Previous studies have also hinted at a role of HIF2 $\alpha$ in regulating $\mathrm{T}_{\mathrm{E}}$ functions (13). Though we found that EPAS1 (which encodes for HIF2 $\alpha$ ) mRNA expression was higher in $\mathrm{T}_{\text {EXP }}$ cells than in PB-Ts, it was expressed at very low levels in both cell types $\left(10^{-6}\right.$ fold expression compared with ACTB mRNA) (Supplemental Figure $7 G$ ), and we were unable to detect HIF $2 \alpha$ protein expression in either $\mathrm{T}_{\mathrm{EXP}}$ or PB-Ts by Western blotting (data not shown). Thus, we focused our study on HIF1 $\alpha$ expression for subsequent analysis.

HIF1 $\alpha$ accumulates in normal cells in hypoxia but is also constitutively overexpressed in some tumor cells in normoxia (25, 30), which facilitates aerobic glycolytic metabolism (the Warburg effect) (25). We postulated that HIF1 $\alpha$ may also be overexpressed in $\mathrm{T}_{\mathrm{EXP}}$ and facilitate their metabolism and survival in hypoxia. Accordingly, we found that while HIF1 $\alpha$ accumulated in PB-Ts after antigen stimulation in hypoxia, $\mathrm{T}_{\mathrm{EXP}}$ showed detectable 
A

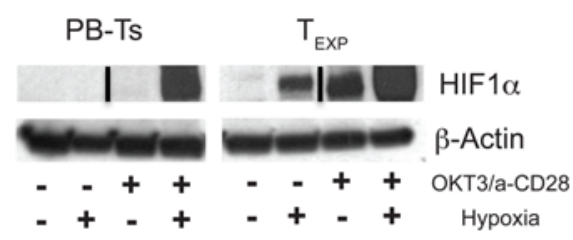

C

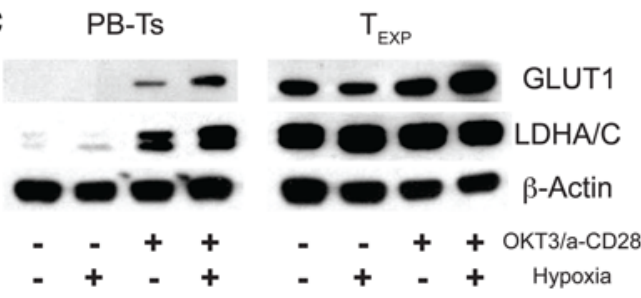

B

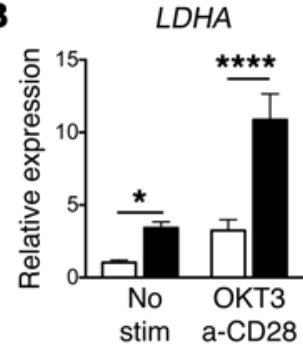

GLUT1

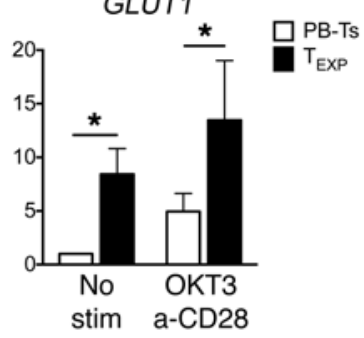

D

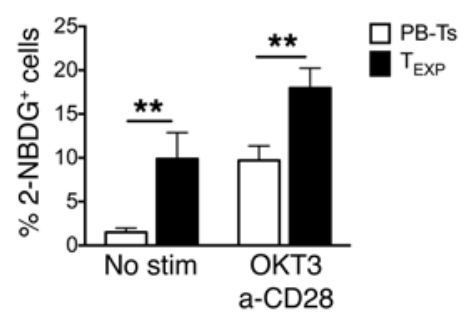

E

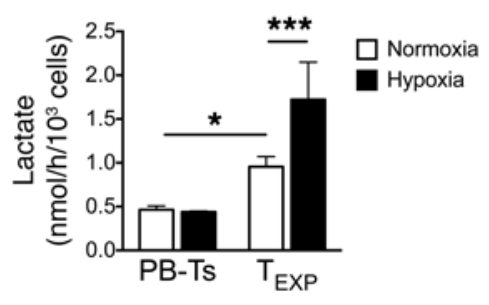

F

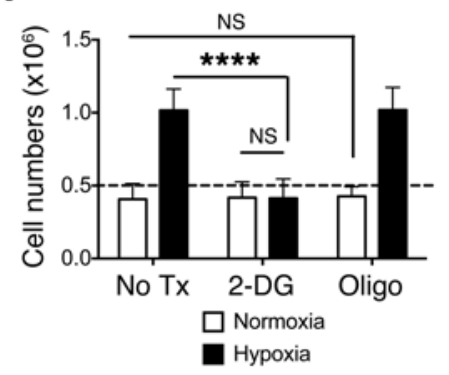

G

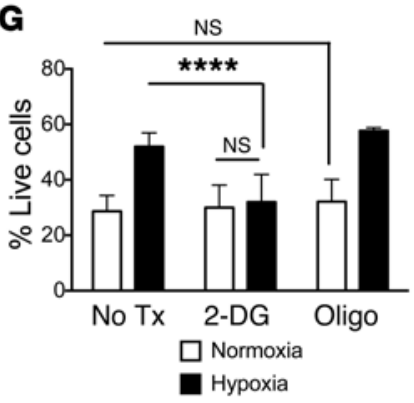

H

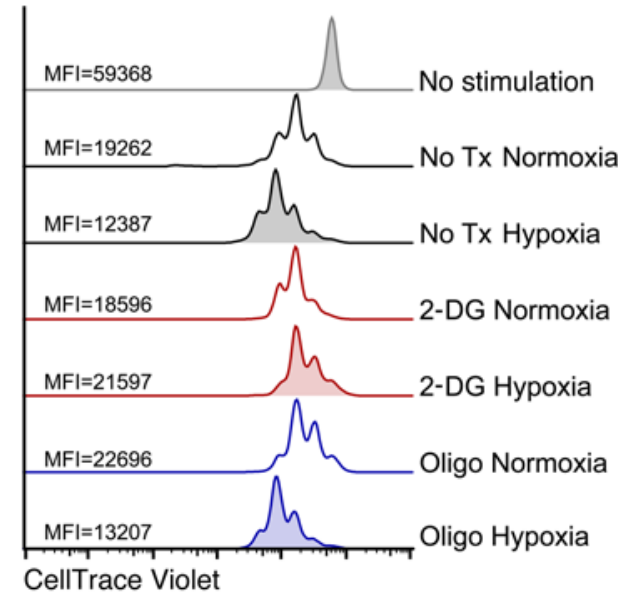

Figure 5. $\mathrm{T}_{\mathrm{EXP}}$ display elevated HIF1 $\alpha$ expression and glycolytic activity. (A) HIF1 $\alpha$ protein expression in PB-Ts and $\mathrm{T}_{\mathrm{EXP}}$ that were unstimulated or stimulated with OKT3/a-CD28 Abs in normoxia or hypoxia for 24 hours. $n=3$. Blot images were acquired from samples run on parallel gels. (B and C) Expression

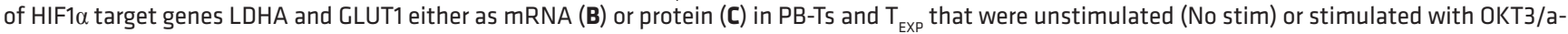
CD28 Abs in normoxia or hypoxia for 24 hours. Transcript expression was normalized against the housekeeping control 18S RNA and then standardized to 1.0 in unstimulated PB-Ts. $n=3 .{ }^{*} P<0.05$ and ${ }^{* * *} P<0.0001$, 2-way ANOVA with Bonferroni's post-hoc analysis. (D) Glucose uptake in PB-Ts and T that were unstimulated or stimulated with OKT3/a-CD28 Abs in hypoxia for 72 hours. $n=3$. ${ }^{* *} P=0.0021$ for unstimulated PB-Ts versus unstimulated $T_{\text {EXP }}$ and ${ }^{* *} P=0.0024$ for stimulated PB-Ts versus stimulated $\mathrm{T}_{\mathrm{EXP}}$, 2-way ANOVA with Bonferroni's post-hoc analysis. (E) Lactate secretion by unstimulated PB-Ts and $T_{\text {EXP }}$ in normoxia and hypoxia for 6 hours. $n=3 .{ }^{*} P=0.018$ and ${ }^{* * *} P=0.0003,2$-way ANOVA with Bonferroni's post-hoc analysis. ( $\left.\mathbf{F}-\mathbf{H}\right) T_{\text {EXP }}$ were untreated (No Tx) or exposed to $1 \mathrm{mM} 2$-DG or $50 \mathrm{nM}$ oligomycin (Oligo) after stimulation with OKT3/a-CD28 Abs in normoxia or hypoxia. Cell counts (F) and cell viability (G) were determined 72 hours after activation. $n=13$ for untreated and 2-DG, and $n=5$ for oligomycin. ${ }^{* * *} P<0.0001,2$-way ANOVA with Bonferroni's post-hoc analysis. (H) CellTrace Violet dilution of labeled $\mathrm{T}_{\mathrm{EXP}} 72$ hours after stimulation. $n=6$. Error bars indicate SD.

HIF1 $\alpha$ expression in hypoxia without TCR/CD28 stimulation, and expression increased after stimulation in either hypoxia or normoxia (Figure 5A). Since we could not acquire a sufficient number of freshly isolated $\mathrm{T}_{\mathrm{EM}}$ for immunoblot analysis via FACS sorting (Supplemental Figure 5), we compared HIF1 $\alpha$ expression in FACSsorted $\mathrm{T}_{\mathrm{N}}, \mathrm{T}_{\mathrm{CM}}$, and $\mathrm{T}_{\mathrm{EM}}$ by quantitating intracellular HIF1 $\alpha$ staining and flow cytometry. Using this approach, HIF1 $\alpha$ protein expression dynamics mirrored the responses to the hypoxia and antigen receptor stimulation observed in $\mathrm{T}_{\mathrm{EXP}}$ (Supplemental Figure 8, A and B). We thus applied our staining protocol to FACS-sorted $\mathrm{T}_{\mathrm{N}}, \mathrm{T}_{\mathrm{CM}}$, and $\mathrm{T}_{\mathrm{EM}}$ subsets. Notably, HIF1 $\alpha$ only accumulated after activation in hypoxia in $\mathrm{T}_{\mathrm{N}}$ and $\mathrm{T}_{\mathrm{CM}}$ (Supplemental Figure 8, C and $\mathrm{D}$ ), while $\mathrm{T}_{\mathrm{EM}}$ showed elevated HIF1 $\alpha$ expression in hypoxia without stimulation, which was further increased after activation, as observed in $\mathrm{T}_{\text {EXP }}$ (Supplemental Figure 8, C and D). These data suggest that the differential HIF1 $\alpha$ expression in $\mathrm{T}_{\mathrm{EXP}}$ and $\mathrm{PB}$-Ts represents a bona fide difference between $\mathrm{T}_{\mathrm{N}} / \mathrm{T}_{\mathrm{CM}}$ and $\mathrm{T}_{\mathrm{EM}}$ subsets.

Consistent with HIF1 $\alpha$ expression, the HIF1-targeted glycolytic genes GLUT1 and LDHA were expressed at higher levels in $\mathrm{T}_{\text {EXP }}$ compared with levels detected in PB-Ts (Figure 5, B and C). The higher expression of these genes in $\mathrm{T}_{\mathrm{EXP}}$ was associated with more glucose uptake (Figure 5D) and lactate secretion (Figure 5E). Notably, the glycolytic activity in unstimulated $\mathrm{T}_{\mathrm{EXP}}$ was almost comparable to that of activated $\mathrm{PB}-\mathrm{Ts}$, suggesting that $\mathrm{T}_{\mathrm{EM}}$ are imprinted to preferentially 
A

3'-UTR: Ctrl
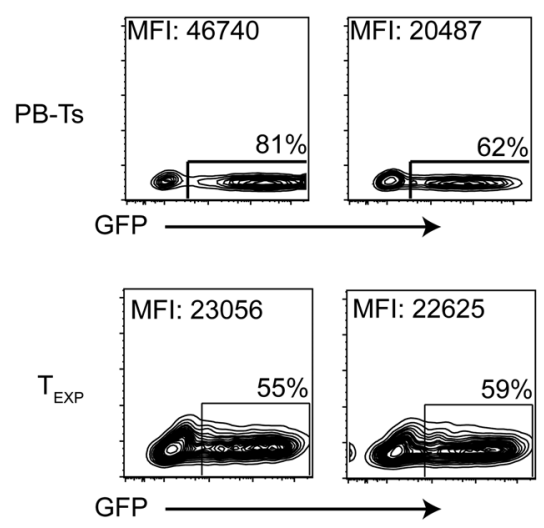

GFP

\section{C}
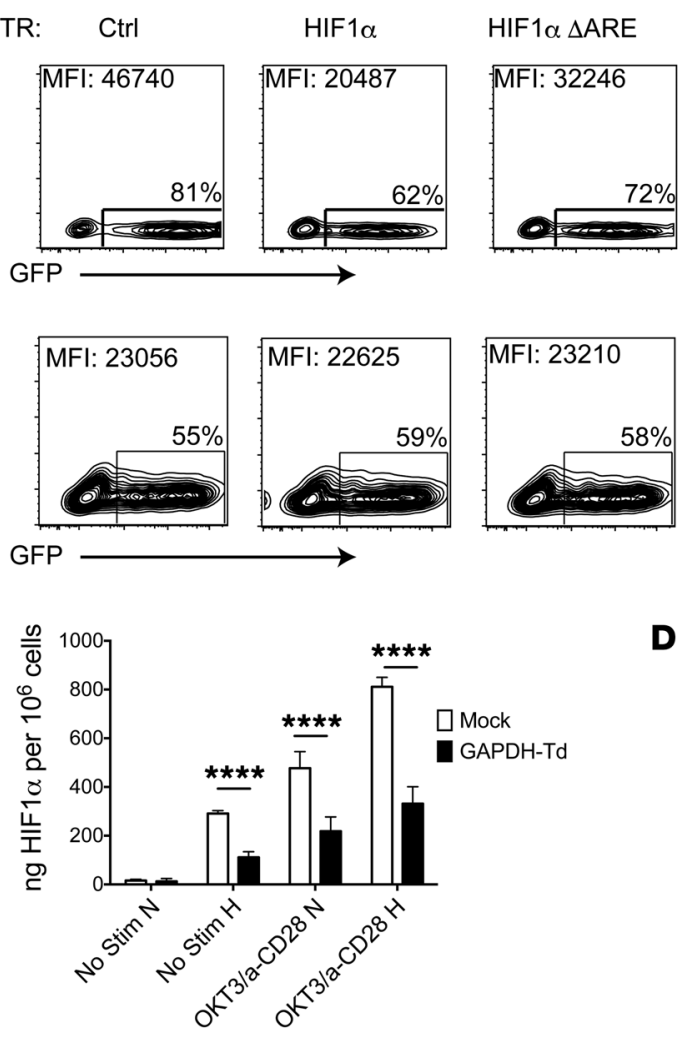

D
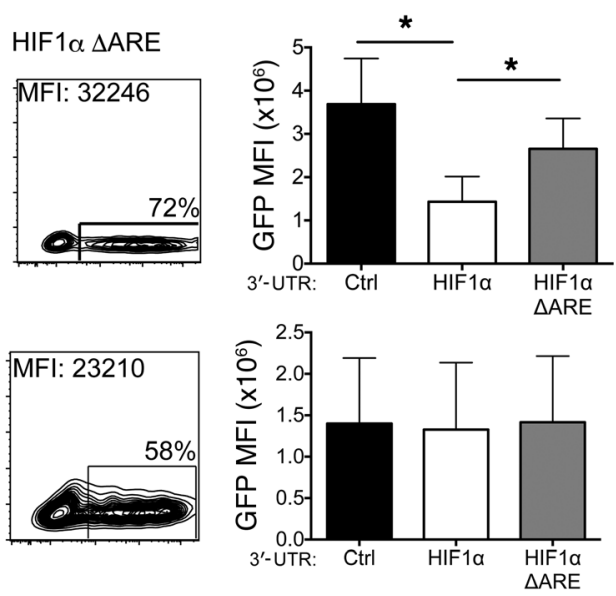

B
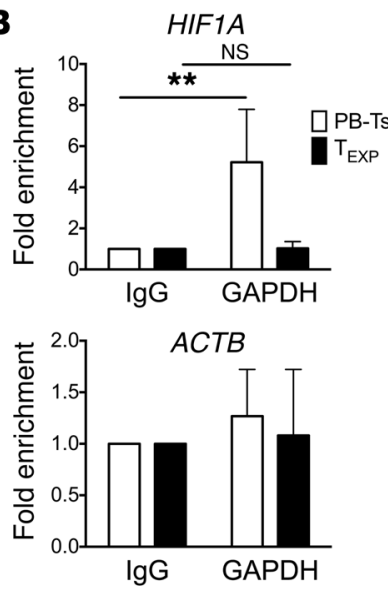
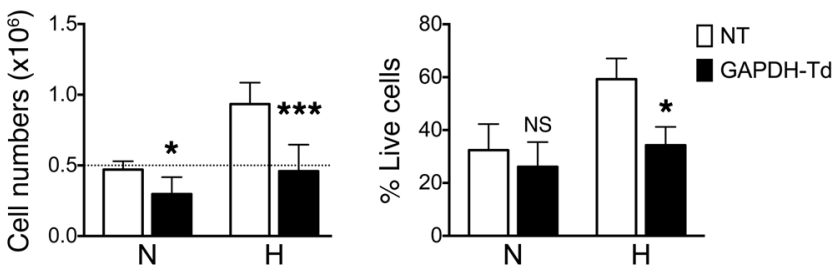

Figure 6. The expression of HIF1a in T cell memory subsets is translationally regulated by GAPDH. (A) A GFP/HIF1A 3'-UTR reporter construct was introduced into PB-Ts and $\mathrm{T}_{\text {EXP }}$ by nucleofection, and GFP expression was measured 8 hours after transfection. The percentages in $\mathbf{A}$ indicate the percentage of GFP ${ }^{+}$ cells. $n=3$ for PB-Ts and $n=3$ for $T_{\text {EXP. }}{ }^{*} P=0.03$, paired Student's $t$ test. (B) GAPDH-specific Abs were used to immunoprecipitate GAPDH from PB-Ts and T EXP. Mouse IgC Ab was used as a control for the IP. The immunoprecipitated mRNA was analyzed by qPCR to quantify HIF1A and ACTB mRNA. Data were normalized against the IgG control pulldown. $n=3 .{ }^{* *} P=0.0081$, 2-way ANOVA with Bonferroni's post-hoc analysis. (C) Quantification of HIF1 $\alpha$ protein expression in mock-transduced $\mathrm{T}_{\mathrm{EXP}}$ or GAPDH-transduced (GAPDH-Td) $\mathrm{T}_{\mathrm{EXP}}$ that were unstimulated or stimulated with OKT3/a-CD28 Abs in normoxia or hypoxia. $n=3$. ${ }^{* * *} P<0.0001,2$-way ANOVA with Bonferroni's post-hoc analysis. (D) Cell counts and cell viability were determined in the $T_{\mathrm{EXP}} 72$ hours after activation. $n=4$. ${ }^{*} P<0.05$ and ${ }^{* * *} P=0.0002,2$-way ANOVA with Bonferroni's post-hoc analysis. Error bars indicate SD. Dotted line indicates the starting cell number.

use glucose metabolism that is similar to that of activated $\mathrm{T}_{\mathrm{N}}(17)$. The high glycolytic activity of $\mathrm{T}_{\mathrm{EXP}}$ is critical for their functionality in hypoxia, given that partial inhibition of glycolysis in $\mathrm{T}_{\mathrm{EXP}}$ using low doses of the competitive glycolytic inhibitor 2-deoxyglucose (2-DG) was sufficient to abolish the enhanced viability and proliferation of $\mathrm{T}_{\mathrm{EXP}}$ in hypoxia (Figure 5, F-H, and Supplemental Figure 9, A-C). The mitochondrial inhibitor oligomycin, however, did not affect the proliferation or survival of $\mathrm{T}_{\mathrm{EXP}}$ in either normoxic or hypoxic conditions, which suggests that, like tumor cells (25), $\mathrm{T}_{\mathrm{EXP}}$ may have intrinsically lower or dispensable mitochondrial functions that allow them to overcome the severe impairment in hypoxia of ATP synthesis from OXPHOS. In contrast, oligomycin suppressed the expansion and viability of PB-Ts in normoxia but did not contribute synergistically to the inhibitory effects of hypoxia (Supplemental Figure 9, D-F). These latter observations suggest that the reduction of $\mathrm{PB}-\mathrm{T}$ proliferation and survival in hypoxia may be attributed to a mechanism related to the inhibition of mitochondrial function. In summary, our data demonstrate that $\mathrm{T}_{\mathrm{EXP}}$ express high levels of HIF1 $\alpha$ and display a glycolysiscentric metabolism, which is critical for their functionality in hypoxia.

Differential expression of HIF1 $\alpha$ in T cell memory subsets is translationally regulated by GAPDH. To mechanistically explain the distinct hypoxia response patterns in $\mathrm{T}$ cell memory subsets, we investigated the molecular programs that regulate HIF1 $\alpha$ expression in both $\mathrm{T}_{\mathrm{EXP}}$ and PB-Ts. The steady-state expression of the mRNA transcript encoding HIF1 $\alpha$ was comparable between unstimulated $\mathrm{T}_{\text {EXP }}$ and $\mathrm{PB}-\mathrm{Ts}$ and was similarly induced after $\mathrm{T}$ cell activation (Supplemental Figure 10A). These results excluded a primarily transcriptional regulatory mechanism underling the differential relative expression of HIF1 $\alpha$ protein in these 2 populations of cells. Inhibition of proteasomal degradation did not normalize HIF1 $\alpha$ protein expression in $\mathrm{T}_{\mathrm{EXP}}$ or PB-Ts and only minimally increased HIF1 $\alpha$ levels in unstimulated PB-Ts, as measured by quantitative HIF1 $\alpha$ ELISA (Supplemental Figure 10B), suggesting that differential proteasome degradation is not sufficient to explain the observed differences in HIF1 $\alpha$ protein expression in $\mathrm{PB}-\mathrm{Ts}$ and $\mathrm{T}_{\mathrm{EXP}}$. Together, these data suggest the possibility that translation of the HIF1A mRNA transcript might be inhibited in PB-Ts. Because the translation of HIF1A in T cells is facilitated by IL-2 signaling and the PI3K/mTOR pathway $(12,33)$, we evaluated the phosphorylation status of mTOR and its effector $\mathrm{S} 6$ kinase in both normoxia and hypoxia in $\mathrm{T}_{\mathrm{EXP}}$ and PB-Ts, but did not find a significant difference (Supplemental Figure 10C).

Messenger RNAs containing cis-acting adenylate-uridylate-rich elements (AREs) in their 3'-UTRs are often targets of RNA-binding proteins that regulate their stability $(34,35)$. In situ analysis revealed 
7 "AUUUA" pentamer AREs and 3 "UUAUUUAUU" nonamer AREs within the HIF1A 3'-UTR (Supplemental Figure 11A). Many of these AREs are highly evolutionarily conserved among different species (3'UTR analyzed using the AREsite database; http://nibiru.tbi.univie. ac.at/AREsite2/welcome) (36), suggesting that these elements may be pivotal in HIF1A mRNA regulation. We thus performed a 3'-UTRbased reporter assay to determine whether the HIF1A 3'-UTR alone is adequate to confer translational regulation in T cells. The HIF1A 3'-UTR specifically reduced the reporter expression in PB-Ts but not in $\mathrm{T}_{\mathrm{EXP}}$ (Figure 6A). Notably, the reduction in reporter expression by HIF1A 3'-UTR in PB-Ts was partially reverted when the AREs were mutated. These data suggest a specific translational regulation of HIF1A mRNA mediated by the AREs in its 3'-UTR in PB-Ts, but not in $\mathrm{T}_{\mathrm{EXP}}$. Importantly, we found that the insensitivity to translational suppression by the HIF1A 3'-UTR of $\mathrm{T}_{\text {EXP }}$ may be attributed to their high glycolytic activity. $\mathrm{T}_{\mathrm{EXP}}$ with dampened glycolysis by 2-DG showed reduced reporter expression with the HIF1A 3'-UTR compared with control 3'-UTR or the HIF1A 3'-UTR ARE mutant (Supplemental Figure 11B). These data suggest a link between the regulation of HIF1a expression via its 3'-UTR and glycolytic status in T cells.

The glycolytic enzyme GAPDH can function as an RNA-binding protein $(37,38)$ and can negatively regulate the translation of IL-2 and IFN- $\gamma$ in activated T cells via specific binding to AREs within the mRNAs encoding these gene products $(38,39)$. Notably, this translational regulation only occurs in $\mathrm{T}$ cells with low glycolytic activity when GAPDH is not engaged in its main function as a glycolytic enzyme (39). Thus, we tested whether GAPDH may also act as a suppressor for HIF1A mRNA translation, preferentially in PB-Ts that have less active glycolysis as compared with $\mathrm{T}_{\text {EXP }}$ that have high glycolytic activity. We performed RNA IP assays using GAPDH Ab and found a greater than 5-fold enrichment of HIF1A mRNA in the $\alpha$-GAPDH Ab immunoprecipitate compared with the IgG control immunoprecipitate in PB-Ts (Figure 6B). In contrast, no enrichment of HIF1A mRNA was observed in $\mathrm{T}_{\text {EXP. }}$. Furthermore, we did not find enrichment of $\beta$-actin (ACTB) mRNA in either $\mathrm{T}_{\mathrm{EXP}}$ or PB-Ts, suggesting that the binding of GAPDH to HIF1A mRNA is specific.

To further demonstrate the critical role of GAPDH in suppressing HIF1 $\alpha$ protein expression, we overexpressed GAPDH in $\mathrm{T}_{\text {EXP }}$ (Supplemental Figure 12, A and B) and found that HIF1 $\alpha$ protein, but not mRNA, expression was reduced (Figure 6C, Supplemental Figure 12C). Reduction of HIF1 $\alpha$ in $\mathrm{T}_{\text {EXP }}$ by GAPDH overexpression did not impair the differentiation of $\mathrm{T}_{\mathrm{EXP}}$ (Supplemental Figure 12D), but caused a reduction of the glycolytic activity, cell expansion, survival, and proliferation of $\mathrm{T}_{\mathrm{EXP}}$ in hypoxia (Figure 6D and Supplemental Figure 12, E and F). Notably, using the in vivo model we developed (shown in Figure 4), we found that CAR.GD2-T in which we overexpressed GAPDH showed significantly reduced proliferation in hypoxic tumor areas compared with that seen in mock CAR.GD2-T (Supplemental Figure 12G). GAPDH is thus a novel regulator for HIF1A mRNA translation and links HIF1 $\alpha$ protein expression to the glycolytic activity in T cell memory subsets.

\section{Discussion}

$\mathrm{T}$ cell activation relies not only on TCR and costimulatory signals but also on environmental inputs including $\mathrm{O}_{2}$ availability. Here, we demonstrate dichotomous roles of hypoxia on human $\mathrm{T}$ cell subsets. Specifically, while $\mathrm{T}_{\mathrm{N}}$ and $\mathrm{T}_{\mathrm{CM}}$ are suppressed in hypoxia, $\mathrm{T}_{\mathrm{EM}}$ show elevated proliferation, survival, and cytotoxic activity. Therefore, hypoxia may serve as both a tolerance mechanism for $\mathrm{T}_{\mathrm{N}}$ and $\mathrm{T}_{\mathrm{CM}}$ in secondary lymphoid organs by preventing excessive proliferation and a license mechanism for $\mathrm{T}_{\mathrm{EM}}$ in inflamed or malignant tissues by facilitating proliferation and effector functions.

$\mathrm{T}_{\mathrm{N}}, \mathrm{T}_{\mathrm{CM}}$, and $\mathrm{T}_{\mathrm{EM}}$ have highly specified functional distinctions, and thus it is intuitive that low $\mathrm{O}_{2}$ supply may be perceived differently by these subsets. In particular, the assumption that hypoxia impairs T-cell-mediated immune responses $(40,41)$ does not take into account the fact that hypoxia is present in almost all pathological conditions in which immune cells must execute essential protective functions. We have found that $\mathrm{T}_{\mathrm{EM}}$, but not $\mathrm{T}_{\mathrm{N}}$ or $\mathrm{T}_{\mathrm{CM}}$, react to low $\mathrm{O}_{2}$ supply by proliferating. Of note, although $\mathrm{T}_{\mathrm{EM}}$ have a proliferative potential inferior to that of $\mathrm{T}_{\mathrm{N}}$ and $\mathrm{T}_{\mathrm{CM}}$ upon stimulation in normoxia (14), this deficiency is corrected in hypoxia, in which $\mathrm{T}_{\mathrm{EM}}$ divisions are almost comparable to those of $\mathrm{T}_{\mathrm{N}}$ and $\mathrm{T}_{\mathrm{CM}}$ in normoxia. These findings suggest that $\mathrm{T}_{\mathrm{EM}}$ are not intrinsically restricted in their proliferative capacity compared with $\mathrm{T}_{\mathrm{N}}$ or $\mathrm{T}_{\mathrm{CM}}$ but require additional environmental input to fully exploit it. Alternatively, the $\mathrm{O}_{2}$-rich condition may pose an inhibitory effect on $\mathrm{T}_{\mathrm{EM}}$, possibly through the generation of ROS, which is relieved in hypoxia $(25,42)$. Further studies are needed to elucidate these mechanisms.

The enhanced functions of $\mathrm{T}_{\mathrm{EM}}$ in hypoxia correspond to a "hypoxia adaptation signature" with higher expression of the master hypoxia transcriptional regulator HIF1 $\alpha$ and increased activity and dependency of aerobic glycolysis in $\mathrm{T}_{\mathrm{EM}}$ compared with that in $\mathrm{T}_{\mathrm{N}}$ and $\mathrm{T}_{\mathrm{CM}} \cdot \mathrm{T}_{\mathrm{EM}}$ are insensitive to mitochondrial inhibition, while a reduction of glycolytic activity abrogates their functional advantage in hypoxia. Of note, neutrophils and macrophages, which are immune cells specialized to function within hypoxic inflammatory sites, show similar glycolysis-dominant metabolic profiles $(6,43)$, suggesting that the observed hypoxia adaptation signature in $\mathrm{T}_{\mathrm{EM}}$ may represent an evolutionarily conserved pathway for cells that function in low- $\mathrm{O}_{2}$ conditions. This is further supported by findings in cancer cells that exclusively rely on aerobic glycolysis for their growth (25) and the overexpression of HIF1 $\alpha$ in many solid tumors, even in the absence of related genetic mutations $(2,30)$.

How and whether HIF1 $\alpha$ contributes to the regulation of T cell responses remains controversial. Earlier studies demonstrated that targeted deletion of HIF1 $\alpha$ in T cells enhanced their proliferation and antibacterial responses, suggesting a negative regulatory function of HIF1 $\alpha$ in $\mathrm{T}$ cells that might protect tissues from immune damage (41). In contrast, recent findings suggest that HIF1 $\alpha$ is critical for the differentiation and function of $\mathrm{T}_{\mathrm{E}}(12,13)$. These discrepancies can be attributed to differences in infection models and uncharacterized roles of HIF1 $\alpha$ during the development of T cells in HIF1 $\alpha$-deleted mice. Our data in human T cells are consistent with those from more recent studies in mice showing that HIF1 $\alpha$ and hypoxia promote $\mathrm{T}_{\mathrm{EM}}$ functions $(12,13)$. This functional correlation is further supported by the mechanistic link we have identified between HIF1 $\alpha$ expression and glycolysis. We discovered that HIF1 $\alpha$ can be regulated at the translational level by the glycolytic enzyme GAPDH. In $\mathrm{T}_{\mathrm{N}}$ and $\mathrm{T}_{\mathrm{CM}}$, GAPDH is disengaged from the glycolytic pathway, since these cells are characterized by low glycolytic activity, and GAPDH can bind to the AREs in the 3'-UTR of HIF1A mRNA, suppressing its translation. In contrast, GAPDH binding is absent in $\mathrm{T}_{\mathrm{EM}}$, which have enhanced glycolytic 
activity, and GAPDH is fully engaged in glycolysis. This role of GAPDH mimics the mechanism that was previously observed for IFNG mRNA, which is also regulated at the translational level by GAPDH in a glycolysis-related manner (39). Unfortunately, we could not perform RNA IP experiments using freshly isolated $\mathrm{T}_{\mathrm{EM}}$ because of the scarceness of this subset in the peripheral blood, and thus we cannot formally prove that GAPDH binds to HIF1A mRNA in $\mathrm{T}_{\mathrm{EM}}$. Although we also acknowledge that $\mathrm{T}_{\mathrm{EXP}}$ cannot be considered identical to $\mathrm{T}_{\mathrm{EM}}$ freshly isolated from the peripheral blood, we showed that both $\mathrm{T}_{\mathrm{EM}}$ and $\mathrm{T}_{\mathrm{EXP}}$ have similar upregulation of HIF1 $\alpha$ protein expression and phenotypic behavior in hypoxia. It is also likely that additional mechanisms may also contribute to the enhanced expression of HIF $1 \alpha$ in $\mathrm{T}_{\mathrm{EM}}$. Our findings also suggest a positive feedback loop between HIF1 $\alpha$ expression and glycolysis in T cells. While the inhibition of HIF1 $\alpha$ expression by GAPDH is relieved upon the elevation of glycolytic activity, HIF1 transactivates its target genes that further facilitate glycolysis. When and how this loop is initiated during $\mathrm{T}$ cell differentiation remains to be investigated. Previous studies showed that Myc mediates a glycolytic switch upon activation in $\mathrm{T}_{\mathrm{N}}(17)$. It is thus possible that the effect of Myc is still persisting in T cells differentiating in culture upon stimulation and this could partially explain the glycolytic activity of $\mathrm{T}_{\mathrm{EXP}}$ in steady-state conditions under normoxia.

We believe our experiments using adoptively transferred tumor-specific $\mathrm{T}$ cells also provide significant insight to the field of cancer immunotherapy. The microenvironment in solid tumors is hypoxic due to dysfunctional angiogenesis $(4,25)$. The "hypoxia adaptation signature" we found in $\mathrm{T}_{\mathrm{EM}}$ can be potentially exploited through genetic manipulations in subsets of tumor-specific $\mathrm{T}$ cells to enhance their functions in hypoxic tumor sites. On the other hand, the tumor microenvironment is also deprived of glucose and other nutrients because of the high metabolic rate of tumor cells (44). T cells must compete with tumor cells for glucose to support their effector functions and proliferation in hypoxia, and $\mathrm{T}$ cells in metabolite-restricted conditions express high levels of the inhibitory receptor programmed death 1 (PD1) and produce less IFN- $\gamma$ upon activation (39); thus, strategies aimed at enhancing the capacity of $\mathrm{T}$ cells to compete for glucose may enhance their antitumor effects.

Overall, we believe our findings redefine the role of hypoxia in regulating $\mathrm{T}$ cell responses, especially in pathological peripheral tissues. The translational regulation of HIF1 $\alpha$ by the glycolytic enzyme GAPDH represents a novel mechanism in the control of $\mathrm{T}$ cell functions via cellular metabolism.

\section{Methods}

\section{Cell line}

The CHLA-255 neuroblastoma cell line was provided by L.S. Metelitsa of Baylor College of Medicine (45) and was maintained in IMDM (Gibco) supplemented with 10\% FBS (HyClone) and 2 mM Glutamax (Invitrogen). The LA-N-1 neuroblastoma cell line was obtained from M. Brenner at Baylor College of Medicine. The LA-N-1 cell line and EBV-transformed lymphoblastoid cell lines (LCLs) were cultured in RPMI 1640 (HyClone) supplemented with 10\% FBS (HyClone) and $2 \mathrm{mM}$ Glutamax (Invitrogen). All cell lines were routinely tested for mycoplasma and for surface expression of target antigens.

\section{Isolation of human peripheral blood T cells and expansion of $\mathrm{T}_{\mathrm{EM}}$} Human PBMCs were obtained from healthy volunteer donors (Gulf Coast Regional Blood Center) by Ficoll, and peripheral blood T cells were isolated by magnetic sorting using a pan-T cell selection kit from Miltenyi Biotec. $\mathrm{T}_{\mathrm{EM}}$ were expanded by stimulation with plate-bound OKT3 $(1 \mathrm{ng} / \mathrm{ml})$ and CD28 Abs (BD Biosciences) and then cultured in media containing $45 \%$ Click's media (Irvine Scientific); 45\% RPMI 1640 (HyClone); 10\% FBS (HyClone); 1\% L-glutamine (Invitrogen); and $100 \mathrm{U} / \mathrm{ml}$ IL-2 (Teceleukin) for 10 to 12 days. For CAR-modified $\mathrm{T}_{\mathrm{EM}}$, stimulated PBMCs were transduced with a $\gamma$ retroviral vector encoding a second-generation (CD28) CAR targeting the GD2 antigen (CAR.GD2) and maintained in $50 \mathrm{U} / \mathrm{ml} \mathrm{IL-2} \mathrm{for} 10$ days.

\section{Cell sorting}

For isolation of $\mathrm{T}_{\mathrm{N}}, \mathrm{T}_{\mathrm{CM}}$, and $\mathrm{T}_{\mathrm{EM}}$ subsets, peripheral blood $\mathrm{T}$ cells were labeled with FITC-conjugated anti-CCR7 (R\&D Systems) and APC-conjugated anti-CD45RA Abs (BD Biosciences). CCR $7^{+} \mathrm{CD} 45 \mathrm{RA}^{+}$ (naive), $\mathrm{CCR}^{+} \mathrm{CD} 45 \mathrm{RA}^{-}$(central memory), and $\mathrm{CCR} 7^{-} \mathrm{CD} 45 \mathrm{RA}^{-}$ (effector memory) subsets were then sorted by FACS. The purity of sorted subsets was greater than $95 \%$.

\section{In vitro hypoxia incubation}

Most of the hypoxia experiments were conducted using the hypoxia incubator (Thermo Scientific). For protein analysis and other timesensitive experiments, a hypoxia glove box (Coy Lab Products) was used so that protein extraction was performed under hypoxic conditions. To activate $\mathrm{T}$ cells in hypoxia, cells were preincubated in hypoxia $\left(1 \% \mathrm{O}_{2}\right)$ for 24 hours and activated with plate-bound OKT3/a-CD28 Abs. Anti-CAR.GD2 Ab 1A7 was used to activate CAR.GD2-T cells.

\section{Flow cytometry}

Cells were immunostained and acquired on a BD FACSCalibur, Fortessa and Gallios cytometer (Beckman Coulter). The following fluorophoreconjugated Abs were used for flow cytometric analysis: anti-Ki67 (B56); anti-CD3 (SK7); anti-CD4 (SK3); anti-CD8 (SK1); anti-Fas (DX2); antiCD25 (2A3); anti-CD27 (M-T271); anti-CD28 (CD28.2); anti-CD45RO (UCHL-1); anti-CD62L (DREG-56); anti-CD69 (L78); anti-IL-2 (MQ117H12); anti-IFN- $\gamma$ (B27); anti-TNF- $\alpha$ (MAB11); anti-perforin (8G9); anti-granzyme B (GB11); anti-CD107a (H4A3); anti-CD107b (H4B4); anti-phosphorylated-S6 (anti-p-S6) (N4-41); anti-p-mTOR (O21-404) (all from BD); anti-HIF1 $\alpha$ (514-16; BioLegend); and anti-HP1 (4.3.11.3; Hypoxyprobe). Caspase activity was measured with a Vybrant FAM Poly Caspase Kit (Life Technologies). Flow cytometric data were analyzed by FlowJo software, version 9.3.2 (Tree Star).

\section{${ }^{51} \mathrm{Cr}$ release assay}

The cytotoxic activity of CAR-T was evaluated in a standard 6-hour ${ }^{51} \mathrm{Cr}$ release assay. Normoxia-cultured or hypoxia-cultured LA-N-1 cells were labeled for 1 hour at $37^{\circ} \mathrm{C}$ in $5 \% \mathrm{CO}_{2}$ with ${ }^{51} \mathrm{Cr}$ (PerkinElmer), washed 3 times, and plated at $5 \times 10^{3}$ cells per well with CAR-T to give effector/target (E:T) ratios of 40:1, 20:1, 10:1, and 5:1. After 6 hours of incubation in normoxia or hypoxia, the supernatant was harvested from each well, and chromium release was measured in a gamma counter (PerkinElmer). For each target, spontaneous and maximum release were used to calculate the percentage of specific release, according to the following formula: (experimental cpm - spontaneous $\mathrm{cpm})$ / (maximum cpm - spontaneous cpm). 


\section{In vivo hypoxia mouse model}

Intratumoral injection model. Eight-week-old NSG mice (The Jackson Laboratory) were s.c. engrafted with $1 \times 10^{6} \mathrm{CHLA}-255$ neuroblastoma cells. Twenty-one days after tumor engraftment, $1 \times 10^{7}$ CAR.GD2-T cells or control CAR-T cells (CAR.CD19) were injected intratumorally. Three days after $\mathrm{T}$ cell inoculation, mice were injected with $1.5 \mathrm{mg}$ HP1 per mouse for hypoxia labeling. Sixty minutes after HP1 injection, mice were euthanized and tumor tissues collected for FACS analysis.

Systemic injection model. Eight-week-old NSG mice were s.c. engrafted with a $1 \times 10^{6}$ EBV-transformed human B cell line $\left(\mathrm{CD} 19^{+}\right)$. Twenty-one days after engraftment, $1 \times 10^{7}$ CAR.CD19-T cells were injected i.v. Subsequent hypoxia labeling by HP1 and FACS analysis was performed as described for the intratumoral injection model.

Staining procedure. Single-cell suspensions were prepared from the explanted tumor tissues and surface stained with Viability Dye (catalog 423101; BioLegend), anti-human CD3, anti-human CD45, anti-human CD4, and anti-human CD8. Cells were then fixed, permeabilized, and intracellularly stained with anti-HP1 and anti-Ki67.

\section{Quantitative real-time RT-PCR}

Quantitative real-time PCR (qPCR) was performed using TaqMan Gene Expression Master Mix (Life Technologies). TaqMan qPCR primers for HIF1A, SLC2A1 (GLUT1), LDHA, BCL2, BNIP3, BCL2L1, FAS, FASL, TP53, BAX, BAK, BAD, and EPAS1 (HIF2A), and the loading control 18S RNA were designed by Life Technologies.

\section{Immunoblot analysis}

Proteins were extracted from more than $2 \times 10^{6} \mathrm{~T}$ cells using RIPA buffer (Thermo Fisher Scientific) supplemented with protease and phosphatase inhibitor cocktail (Thermo Fisher Scientific). Protein samples were separated by electrophoresis using 10\% Mini-PROTEAN precast gels (BioRad) and transferred to PVDF membranes (Bio-Rad). Membranes were blocked with 5\% BSA in TBS-5\% Tween and blotted with primary Abs against HIF1 $\alpha$, LDHA/C (Cell Signaling Technologies), and GLUT1 (catalog ab115730; Abcam) and goat anti-rabbit secondary Abs (Santa Cruz Biotechnology Inc.). $\beta$-actin (13E5) was used as a loading control.

\section{Quantitative HIF1 $\alpha$ ELISA}

Proteins were extracted from $2 \times 10^{6} \mathrm{~T}$ cells, and HIF1 $\alpha$ protein levels were measured directly using quantitative HIF1 $\alpha$ ELISA (DYC1935-2; R\&D Systems).

\section{3'-UTR GFP reporter assay}

HIF1 $\alpha$ 3'-UTR or HIF1 $\alpha$ 3'-UTR ARE mutant (T-to-G mutations for all predicted AREs) were cloned after a GFP reporter gene driven by a CMV promoter. A 3'-UTR sequence lacking AREs was used as a control. Reporter vectors were introduced into freshly isolated PB-Ts or $\mathrm{T}_{\mathrm{EXP}}$ via nucleofection (P3 Primary Cell 4D-Nucleofector Kit; Lonza), and GFP expression was determined 8 hours after nucleofection by flow cytometry. As for the 3'-UTR assay on 2-DG-treated $\mathrm{T}_{\mathrm{EXP}}$, the $\mathrm{T}_{\mathrm{EXP}}$ were treated with $1 \mathrm{mM} 2-\mathrm{DG} 48$ hours before nucleofection and cultured for 8 hours with $1 \mathrm{mM}$ 2-DG-containing media after nucleofection.

\section{RNA IP}

Matched donor PB-Ts or $\mathrm{T}_{\text {EXP }}$ were UV-crosslinked with $150 \mathrm{~mJ} / \mathrm{cm}^{2}$ at $254 \mathrm{~nm}$ using a UV Crosslinker (Spectroline) and were lysed with lysis buffer containing $100 \mathrm{~mm} \mathrm{KCl} ; 5 \mathrm{~mm} \mathrm{MgCl} ; 10$ mm HEPES, pH 7.0;
0.5\% Nonidet P-40 detergent supplemented with fresh $1 \mathrm{~mm}$ DTT; 1,000 units/ml RNAsin (Promega); and Mini Protease Inhibitor Cocktail (Thermo Fisher Scientific). The post-nuclear cytosolic content was collected and removed for input samples (10\%). The remaining lysates were immunoprecipitated with $10 \mu \mathrm{g}$ anti-GAPDH Abs (Life Technologies) or with control mouse IgG Abs (The Jackson Laboratory) using a Pierce Crosslink IP Kit (Thermo Fisher Scientific). The rest of the immunoprecipitated complexes were digested with $30 \mu \mathrm{g}$ proteinase $\mathrm{K}$. The immunoprecipitates and input samples were subjected to RNA extraction using TRIzol LS Reagent (Life Technologies). RNA isolates were used for first-strand cDNA synthesis using a SuperScript VILO cDNA Synthesis Kit (Life Technologies). cDNA was used for qPCR quantification, with HIF1 $\alpha$ or $\beta$-actin as a control. The $\mathrm{Ct}$ for each RNA IP sample was normalized to that of total input to account for the differences in sample preparation using the following calculation: $\Delta \mathrm{Ct}$ $($ normalized $)=\mathrm{Ct}($ sample $)-(\mathrm{Ct}$ [input $\left.]-\log _{2}[10]\right)$.

The $\Delta \mathrm{Ct}$ (normalized) of the GAPDH IP sample was further normalized to the IgG IP sample as follows: $\Delta \Delta \mathrm{Ct}=\boldsymbol{\Delta C}$ (normalized) ${ }^{\mathrm{GAPDH}}$ - $\Delta \mathrm{Ct}$ (normalized) ${ }^{\mathrm{IgG}}$.

The immunoprecipitation fold enrichment above the sample-specific background was calculated as follows: fold enrichment $=2^{(-\Delta \Lambda C T)}$.

\section{Statistics}

A paired, 2-tailed Student's $t$ test was used to determine statistically significant differences between 2 samples. When multiple comparison analyses were required, statistical significance was evaluated by ANOVA, followed by Bonferroni's post-hoc analysis. If the data reflected measurement of 1 sample over time or under different conditions, repeated-measures ANOVA was used, followed by Bonferroni's post-hoc analysis. Graph generation and statistical analyses were performed using GraphPad Prism, version 5.0d (GraphPad Software). A $P$ value of less than 0.05 was considered statistically significant.

\section{Study approval}

The present studies in animals were reviewed and approved by the IACUCs of Baylor College of Medicine and the University of North Carolina.

\section{Author contributions}

YX, JR, JRN, and GD designed the experiments. YX, AC, MZ, BS, and JRN conducted the experiments and/or analyzed the data. LSM and JTY provided equipment for the hypoxia experiments. YX and GD wrote the manuscript, and all authors edited the manuscript.

\section{Acknowledgments}

The authors would like to thank Yisong Wan (University of North Carolina) for critical revision of the manuscript and Debra Taxman for editing the manuscript. This work was supported in part by National Cancer Institute (NCI), NIH grant R01 CA142636 and by a Department of Defense and Technology/Therapeutic Development Award (W81XWH-10-10425, to G. Dotti).

Address correspondence to: Gianpietro Dotti, Department of Microbiology and Immunology, University of North Carolina, Lineberger Comprehensive Cancer Center, Marsico Hall, 125 Mason Farm Road, Room 5202, Chapel Hill, North Carolina 27599, USA. Phone: 919.962.8414; E-mail: gdotti@med.unc.edu. 
1. Neumann AK, et al. Hypoxia inducible factor 1 alpha regulates $T$ cell receptor signal transduction. Proc Natl Acad Sci USA. 2005;102(47):17071-17076.

2. Semenza GL. Oxygen homeostasis. Wiley Interdiscip Rev Syst Biol Med. 2010;2(3):336-361.

3. Eltzschig HK, Carmeliet P. Hypoxia and inflammation. N EnglJ Med. 2011;364(7):656-665.

4. Brown JM, Wilson WR. Exploiting tumour hypoxia in cancer treatment. Nat Rev Cancer. 2004;4(6):437-447.

5. Hafler DA, Astier AL. Editorial: T cell regulation by the environment. Front Immunol. 2015;6:229.

6. Palazon A, Goldrath AW, Nizet V, Johnson RS. HIF transcription factors, inflammation, and immunity. Immunity. 2014;41(4):518-528.

7. Ohta A, Diwanji R, Kini R, Subramanian M, Ohta A, Sitkovsky M. In vivo T cell activation in lymphoid tissues is inhibited in the oxygen-poor microenvironment. Front Immunol. 2011;2:27.

8. Conforti L, et al. Hypoxia regulates expression and activity of Kv1.3 channels in T lymphocytes: a possible role in $\mathrm{T}$ cell proliferation. J Immunol. 2003;170(2):695-702.

9. Vallejo AN, Yang H, Klimiuk PA, Weyand CM, Goronzy JJ. Synoviocyte-mediated expansion of inflammatory $\mathrm{T}$ cells in rheumatoid synovitis is dependent on CD47-thrombospondin 1 interaction. J Immunol. 2003;171(4):1732-1740.

10. Wakim LM, Gebhardt T, Heath WR, Carbone FR. Cutting edge: local recall responses by memory $\mathrm{T}$ cells newly recruited to peripheral nonlymphoid tissues. J Immunol. 2008;181(9):5837-5841.

11. Marten NW, Stohlman SA, Zhou J, Bergmann CC. Kinetics of virus-specific $\mathrm{CD} 8{ }^{+}$-T-cell expansion and trafficking following central nervous system infection. J Virol. 2003;77(4):2775-2778.

12. Finlay DK, et al. PDK1 regulation of $\mathrm{mTOR}$ and hypoxia-inducible factor 1 integrate metabolism and migration of $\mathrm{CD} 8^{+} \mathrm{T}$ cells. JExp Med 2012;209(13):2441-2453.

13. Doedens AL, et al. Hypoxia-inducible factors enhance the effector responses of CD8(+) T cells to persistent antigen. Nat Immunol. 2013;14(11):1173-1182.

14. Mueller SN, Gebhardt T, Carbone FR, Heath WR. Memory T cell subsets, migration patterns, and tissue residence. Annu Rev Immunol. 2013;31:137-161.

15. Tanimoto K, Makino Y, Pereira T, Poellinger L. Mechanism of regulation of the hypoxia-inducible factor-1 $\alpha$ by the von Hippel-Lindau tumor suppressor protein. EMBO J. 2000;19(16):4298-4309.

16. Nakamura $\mathrm{H}$, et al. TCR engagement increases hypoxia-inducible factor- $1 \alpha$ protein synthesis via rapamycin-sensitive pathway under hypoxic con- ditions in human peripheral T cells. JImmunol. 2005;174(12):7592-7599.

17. Wang $\mathrm{R}$, et al. The transcription factor Myc controls metabolic reprogramming upon T lymphocyte activation. Immunity. 2011;35(6):871-882.

18. Shi LZ, et al. HIF1 $\alpha$-dependent glycolytic pathway orchestrates a metabolic checkpoint for the differentiation of TH17 and Treg cells. JExp Med. 2011;208(7):1367-1376.

19. Gubser PM, et al. Rapid effector function of memory $\mathrm{CD} 8^{+} \mathrm{T}$ cells requires an immediate-early glycolytic switch. Nat Immunol. 2013;14(10):1064-1072.

20. Conforti L, et al. Hypoxia regulates expression and activity of Kv1.3 channels in T lymphocytes: a possible role in $\mathrm{T}$ cell proliferation. J Immunol. 2003;170(2):695-702.

21. Sallusto F, Lenig D, Förster R, Lipp M, Lanzavecchia A. Two subsets of memory $\mathrm{T}$ lymphocytes with distinct homing potentials and effector functions. Nature. 1999;401(6754):708-712.

22. Ramaswamy M, et al. Specific elimination of effector memory $\mathrm{CD} 4^{+} \mathrm{T}$ cells due to enhanced Fas signaling complex formation and association with lipid raft microdomains. Cell Death Differ. 2011;18(4):712-720.

23. Dotti G, Gottschalk S, Savoldo B, Brenner MK. Design and development of therapies using chimeric antigen receptor-expressing T cells. Immunol Rev. 2014;257(1):107-126.

24. Espinoza I, Peschke P, Karger CP. A model to simulate the oxygen distribution in hypoxic tumors for different vascular architectures. Med Phys. 2013;40(8):081703.

25. Denko NC. Hypoxia, HIF1 and glucose metabolism in the solid tumour. Nat Rev Cancer. 2008;8(9):705-713.

26. Kim H, et al. Engineering human tumor-specific cytotoxic $\mathrm{T}$ cells to function in a hypoxic environment. Mol Ther. 2008;16(3):599-606.

27. Liu D, et al. IL-15 protects NKT cells from inhibition by tumor-associated macrophages and enhances antimetastatic activity. J Clin Invest. 2012;122(6):2221-2233.

28. Ragnum HB, et al. The tumour hypoxia marker pimonidazole reflects a transcriptional programme associated with aggressive prostate cancer. Br JCancer. 2015;112(2):382-390.

29. Varia MA, et al. Pimonidazole: a novel hypoxia marker for complementary study of tumor hypoxia and cell proliferation in cervical carcinoma. Gynecol Oncol. 1998;71(2):270-277.

30. Semenza GL. HIF-1 mediates metabolic responses to intratumoral hypoxia and oncogenic mutations. JClin Invest. 2013;123(9):3664-3671.
31. Helbig L, et al. BAY 87-2243, a novel inhibitor of hypoxia-induced gene activation, improves local tumor control after fractionated irradiation in a schedule-dependent manner in head and neck human xenografts. Radiat Oncol. 2014;9:207.

32. Lee JH, Elly C, Park Y, Liu YC. E3 ubiquitin ligase VHL regulates hypoxia-inducible factor-1 $\alpha$ to maintain regulatory $\mathrm{T}$ cell stability and suppressive capacity. Immunity. 2015;42(6):1062-1074.

33. Nakamura $\mathrm{H}$, et al. TCR engagement increases hypoxia-inducible factor-1 $\alpha$ protein synthesis via rapamycin-sensitive pathway under hypoxic conditions in human peripheral T cells. J Immunol. 2005;174(12):7592-7599.

34. Galban S, et al. RNA-binding proteins HuR and PTB promote the translation of hypoxia-inducible factor 1 $\alpha$. Mol Cell Biol. 2008;28(1):93-107.

35. Kim TW, et al. Tristetraprolin regulates the stability of HIF-1 $\alpha$ mRNA during prolonged hypoxia. Biochem Biophys Res Commun. 2010;391(1):963-968.

36. Gruber AR, Fallmann J, Kratochvill F, Kovarik P, Hofacker IL. AREsite: a database for the comprehensive investigation of AU-rich elements. Nucleic Acids Res. 2011;39(Database issue):D66-D69.

37. Kondo S, et al. Binding of glyceraldehyde-3phosphate dehydrogenase to the cis-acting element of structure-anchored repression in ccn2 mRNA. Biochem Biophys Res Commun. 2011;405(3):382-387.

38. Nagy E, Rigby WF. Glyceraldehyde-3-phosphate dehydrogenase selectively binds AU-rich RNA in the NAD(+)-binding region (Rossmann fold). J Biol Chem. 1995;270(6):2755-2763.

39. Chang $\mathrm{CH}$, et al. Posttranscriptional control of $\mathrm{T}$ cell effector function by aerobic glycolysis. Cell. 2013;153(6):1239-1251.

40. Ohta A, Diwanji R, Kini R, Subramanian M, Ohta A, Sitkovsky M. In vivo T cell activation in lymphoid tissues is inhibited in the oxygen-poor microenvironment. Front Immunol. 2011;2:27.

41. Thiel M, et al. Targeted deletion of HIF-1 $\alpha$ gene in $\mathrm{T}$ cells prevents their inhibition in hypoxic inflamed tissues and improves septic mice survival. PLoS One. 2007;2(9):e853.

42. Hildeman DA, Mitchell T, Kappler J, Marrack P. T cell apoptosis and reactive oxygen species. JClin Invest. 2003;111(5):575-581.

43. Walmsley SR, Whyte MK. Neutrophil energetics and oxygen sensing. Blood. 2014;123(18):2753-2754.

44. Ackerman D, Simon MC. Hypoxia, lipids, and cancer: surviving the harsh tumor microenvironment. Trends Cell Biol. 2014;24(8):472-478.

45. Song L, et al. Oncogene MYCN regulates localization of NKT cells to the site of disease in neuroblastoma. JClin Invest. 2007;117(9):2702-2712. 